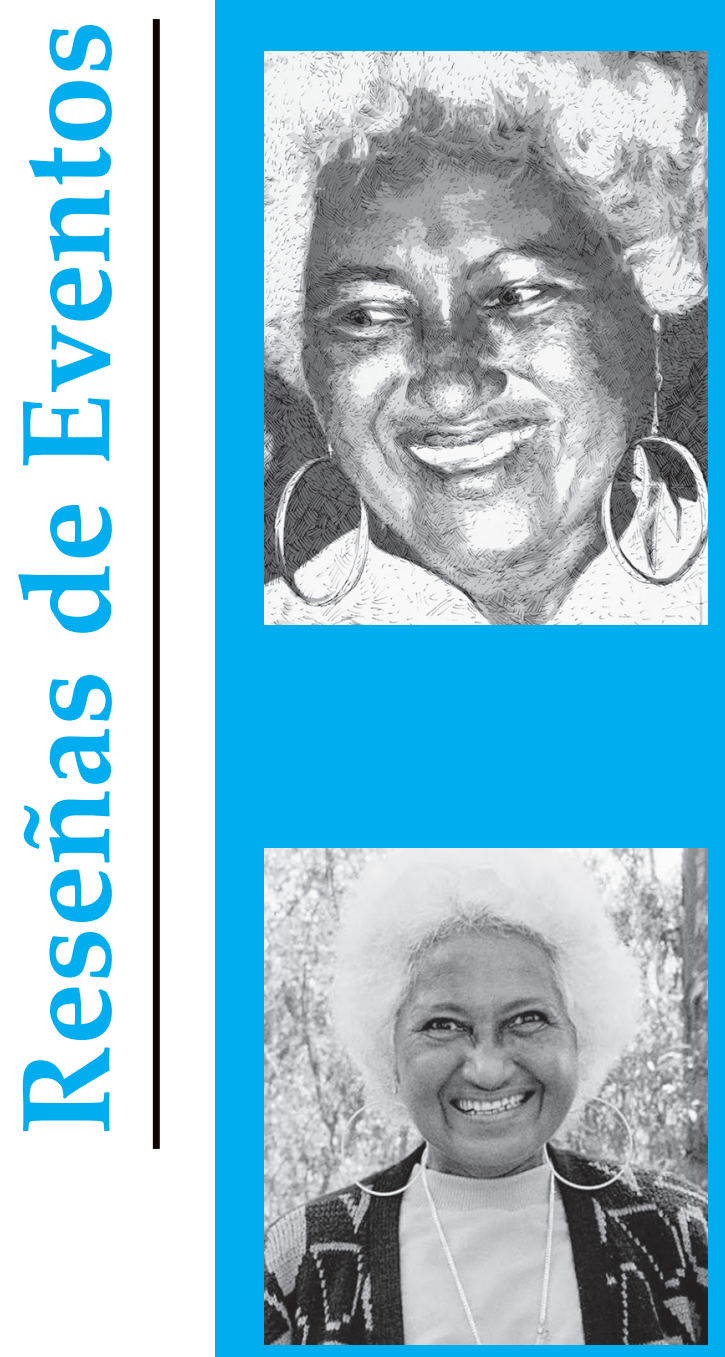

Eventos

\title{
Reseñas Reseñas
}

Reseñas Reseñas de Eventos

Reseñas de Eventos

\section{Reseñas de Eventos}

Reseñas de Eventos

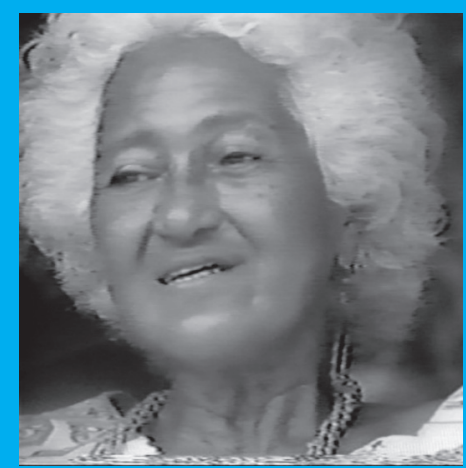





\section{Cátedra HISULA, aprobada por el Consejo Académico de la Universidad Pedagógica y Tecnológica de Colombia 2003.}

Febrero - junio de 2016

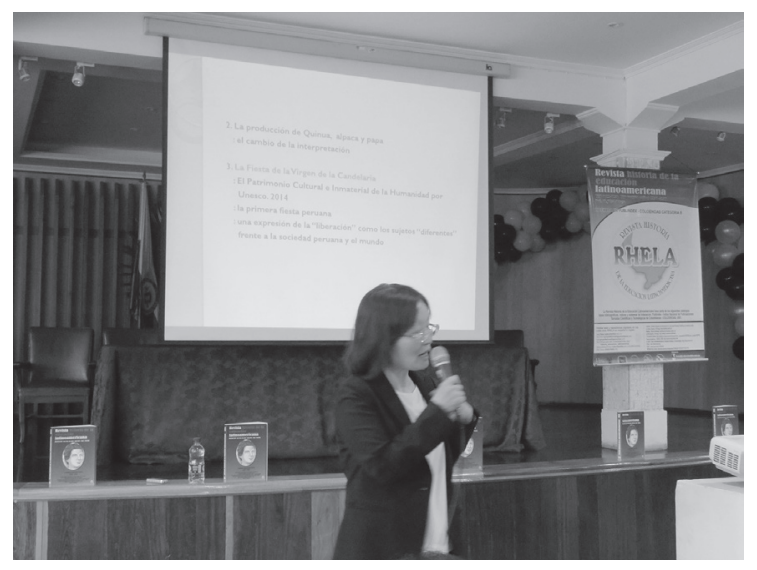

La Cátedra HISULA contó con una prominente participación internacional por parte de investigadores de los grupos HISULA e ILAC de la UPTC durante el primer semestre de 2016. El 24 de febrero se llevó a cabo la primera cátedra del año intitulada: $L a$ educación, paradigmas y profesores: modalidades alternativas. A cargo del Dr. Oscar Hugo López, Ministro de Educación de Guatemala, quien el mismo día, en horas de la tarde, compartió sus perspectivas universitarias con renombrados investigadores como el Dr. Justo Cuño de la Universidad Pablo de Olavide de España, el Dr. José Rubens Lima Jardilino de la Universidad Federal de Ouro Preto de Brasil, y la Dra. Diana Elvira Soto Arango de la Universidad Pedagógica y Tecnológica de Colombia, esto en el marco del segundo evento; El futuro de la Universidad en Guatemala, España, Brasil y Colombia, la asistencia a la cátedra por parte de estudiantes y docentes fue significativa y los investigadores de los grupos HISULA e ILAC ampliaron las discusiones sobre presupuesto, calidad vs. Cobertura, consolidación científica, e internacionalización en sus respectivas asignaturas a partir de las reseñas e inquietudes formuladas por los estudiantes.

El día 25 de febrero, el Dr. Miguel Romero Moret de la Universidad de Guadalajara en México, hizo su aporte con la conferencia: La pedagogía sistémica, transdisciplinaria y dialógica, esta cátedra estuvo dirigida especialmente a los docentes de la escuela de Ciencias Sociales de la UPTC quienes participaron de forma activa y plantearon inquietudes acerca de la articulación de esta propuesta en el contexto colombiano.

Para comenzar el mes de marzo, cuatro destacadas investigadoras de nuestra Alma Máter; Las Dras. Celina Trimiño, Doris Lilia Torres, Gladys Martin, y Wilma Blanco se dieron cita para dar a conocer: El papel de la mujer en la construcción 


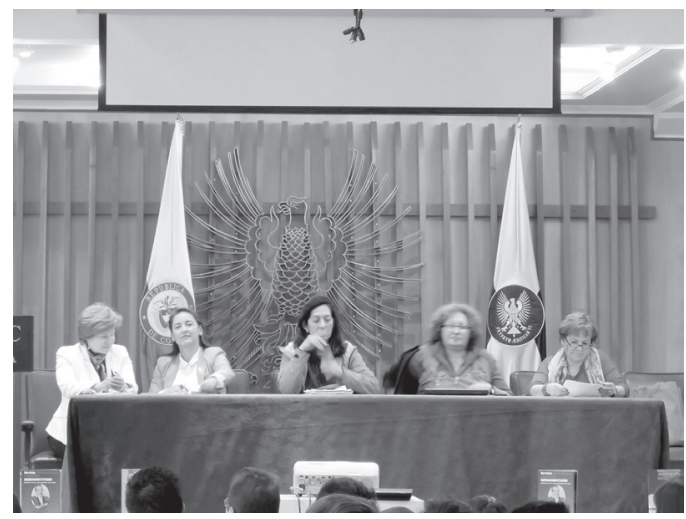

para la paz, sus planteamientos empoderaron la imagen de la mujer universitaria y lideresa que es capaz de mover masas y generar cambios, pero que continúa siendo relegada a pesar de los progresos obtenidos.

Días después, el Dr. Carlos Bauer de la Universidad Uninove de Brasily el Dr. Miguel Beas de la Universidad de Granada en España abordaron la temática: Las pedagogías del siglo XXI, en esta conferencia se compartieron algunas experiencias pedagógicas de los docentes de la UPTC.

Para el día 18 de marzo se planteó la discusión sobre Movimientos estudiantiles en México, Brasil, Chile y Colombia, esta vez el Dr. Carlos Bauer estuvo acompañado por la Dra. Renate Marsiske de la Universidad Nacional Autónoma de México, el Dr. Álvaro Acevedo Tarazona de la Universidad Industrial de Santander, y por el Mg. José Molina Bravo de la Universidad Autónoma de Colombia, todos ellos investigadores con amplia trayectoria en el campo de las revoluciones universitarias. El evento tuvo gran acogida entre la comunidad académica y generó algunos debates sobre los movimientos al interior de la UPTC.

Los días 27, 28, y 29 del mes de abril se realizaron las últimas cátedras HISULA del semestre. En primer lugar, la Doctora en Antropología, Young MiLEE, investigadora del museo de etnología de Osaka, Japón llevó a cabo una interesante conferencia en la cual intentó contrastar La Antropología en Perú y Japón. Inicialmente planteó las nociones de igualdad y equidad desde el indigenismo peruano republicano y la epistemología de la localidad hasta llegar al etnosentido (sentido propio) del pueblo japonés y la construcción de identidades en ambas culturas. La comunidad upetecista acogió las propuestas de MiLEE y algunas reflexiones en torno a la identidad local surgieron durante el evento.

El siguiente día, el Dr. Manuel Álvaro Dueñas, Decano de la Facultad de Educación en la Universidad Autónoma de Madrid, presentó: La didáctica de la Ciencias Sociales, con un público constituido en su mayoría por estudiantes y profesores de filosofía y de la Escuela de Ciencias Sociales. Para culminar con las actividades de divulgación

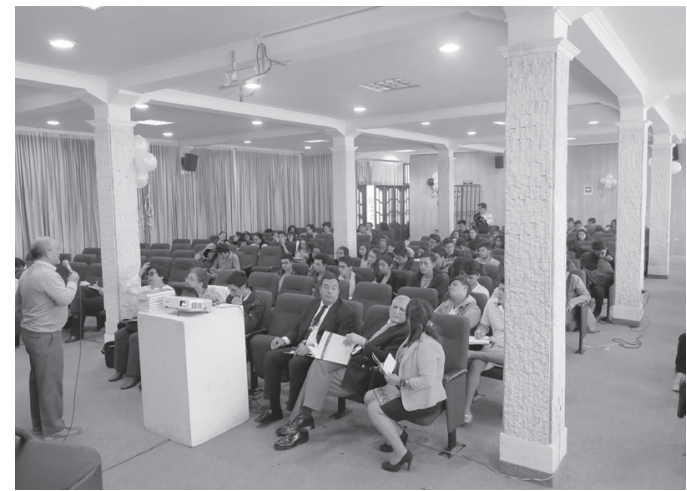


del conocimiento científico, contamos con la participación de dos investigadores españoles y dos locales: la Dra. Fuensanta Hernández de la Universidad de Murcia; el Dr. Antonio Medina de la UNED; el Dr. Nelson López de la Universidad Surcolombiana; y el Dr. Gustavo de la Hoz de la Universidad del Atlántico, quienes expusieron sus percepciones sobre Las pedagogías y la calidad de la educación en Iberoamérica. Siglo XXI. Este cierre permitió retomar algunos asuntos planteados en la cátedra inicial sobre el futuro de la universidad y se determinó que el gran reto sigue siendo la calidad en términos de formación y actualización docente, presupuesto e internacionalización del conocimiento y creación de redes académicas.

Elaboró: María Camila Buitrago Cabrera

Grupo de investigacion HISULA

\section{Conversatorio: Didáctica de las Ciencias Sociales \\ Cátedra HISULA}

Tunja - 28 de abril de 2016

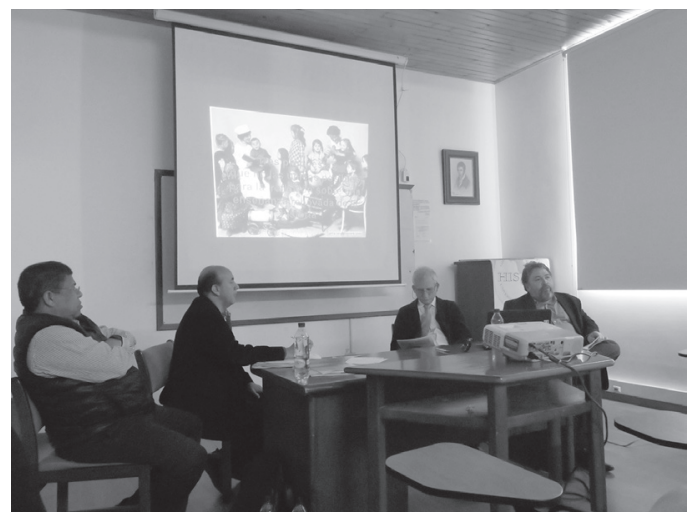

La Cátedra "Historia y Prospectiva de la Universidad Latinoamericana - HISULA", dentro de las actividades de extensión a la comunidad Upetecista desarrolló el Panel: "Didáctica de las Ciencias Sociales" en las instalaciones de la Escuela de Ciencias Sociales, en el marco de la VII jornada académica del Doctorado en Ciencias de la educación RUDECOLOMBIA-

CADE-UPTC. Este evento académico contó con la participación de investigadores de tres universidades: Dr. Manuel Antonio Dueñas, actual decano de la Facultad de Formación del Profesorado y Educación de la Universidad Autónoma de Madrid, España. Dr. Antonio Medina de la Universidad Nacional de Educación a Distancia - Agencia Nacional de Evaluación de la Calidad y Acreditación (UNED-ANECA) de España. Por la Universidad Pedagógica y Tecnológica de Colombia el Dr. Jairo Padilla, docente del área de pedagogía del programa de Ciencias Sociales. El evento estuvo coordinado por el Dr. William Pacheco Vargas con el apoyo del joven investigador Camilo Becerra del grupo de investigación HISULA. 
El Dr. Manuel Álvaro Dueñas, parte de la siguiente reflexión: a la educación secundaria, llega docentes que no tienen vocación ni identidad profesional. Por ello la didáctica la centra desde una aproximación disciplinar, como la historia o la geografía. De ahí que se requiere es una formación desde la psicología del aprendizaje, pues el docente debe saber cómo

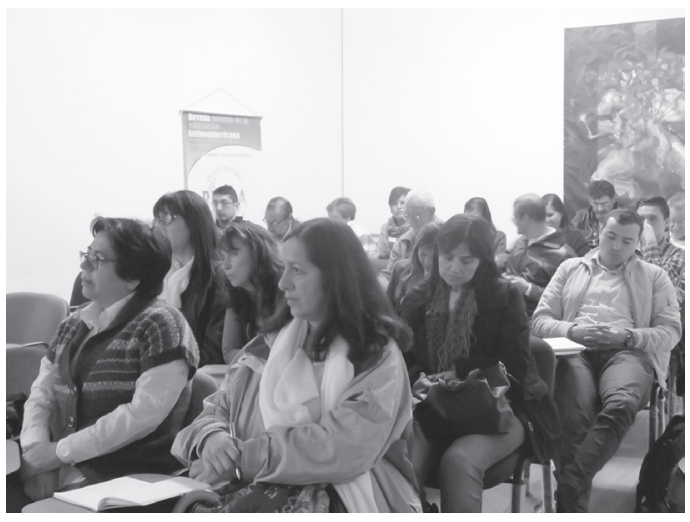
enfrentarse a cada edad y cada concepto. Para enseñar Ciencias Sociales se debe saber cómo integrar los diferentes temas al currículo; en la práctica las asignaturas se fragmentan, no hay integración en los programas en las facultades, en la formación alguien tiene que hacerlo. El diseño de estrategias didácticas requiere una reflexión previa que piense qué conocimiento específico se requiere para enseñar, por qué historia, geografía son auxiliares de la didáctica. Lo que lo lleva a la pregunta: ¿Las Ciencias Sociales o determinada disciplina sirve para que el niño comprenda críticamente su realidad y conozca el mundo? Por ello el currículo es una decisión política de los contenidos que convienen. De ahí que se pregunte nuevamente: ¿Si hay contenidos en Colombia que en el posacuerdo van a permitir hacer tránsito a una sociedad para la paz? Finalmente, considera que la enseñanza de las Ciencias Sociales, debe tener una posición crítica, que discrimine la información que es diferente a conocimiento, entienda los mecanismos de la conformación en las sociedades tecnologizadas, hacer comprensible fenómenos cada vez más visibles en las sociedades en las que viven los jóvenes, ciudadanos y ciudadanas. Ejemplo, la presencia de la violencia de género se debe a la falla del diseño didáctico, por ello se requiere que varios especialistas compartan las asignaturas.

El Dr. Antonio Medina, se pregunta: ¿Si un docente para su formación estudia por la mañana geografía e historia y por la tarde pedagogía? Lo que se requiere en este momento, es resolver desde las fronteras de la investigación didáctica el saber social. El quehacer didáctico le ofrece la oportunidad de disfrutar y adquirir el conocimiento, siempre y cuando, si el docente es un investigador y quiera desarrollar el saber social. La epistemología de la didáctica de las Ciencias Sociales, es el eje didáctico en función del saber social. De esta manera, la didáctica es una actividad práctica que parte de identificar un problema, desde una perspectiva, ejemplo: espacial, de la ciudad y del patrimonio, lo formula, justifica, relaciona, proyecta, e integra todos los saberes; investigación que le permite ser un magnífico didacta. La anterior contextualización, le permite afirmar que las clases de humanidades no se pueden convertir en un 
opinadero, pues el estudio de caso desde el punto de vista didáctico les permite a los estudiantes identificar inquietudes relevantes (Del pasado, presente y del futuro) desde la experiencia investigativa que quieran resolver integrando todos los saberes, así la Universidad deja de amparar la inmediatez. El proceso de enseñanza - aprendizaje, que requiere el docente, está en interactuar con sus estudiantes, construyendo ambientes de aprendizaje, pues el futuro no es trabajar solo en forma presencial, sino aprovechando las plataformas, las redes sociales, mediante el Chad, el foro, la video-conferencia, entre otros. Finalmente, la reflexión del Dr. Antonio Medina de la formación didáctica del profesorado, donde al interactuar entre estudiantes y docentes por los nuevos medios, se convierte no solo en práctica sino que se da el salto a la investigación sobre las mismas prácticas. Donde convierte la docencia en un proceso de investigación, porque no tiene sentido enseñar si no se aporta en la transformación de la sociedad.

El Dr. Jairo Padilla, por su parte, presenta una ponencia desde una perspectiva histórica sobre el estado de las Ciencias Sociales en Colombia, donde se pregunta por el significado de la enseñanza y que seguramente lo lleva a determinar los propósitos que ha tenido la formación de educadores en concordancia con los desafíos y retos del país en cada momento del siglo XX, siguiendo la legislación educativa. Lo cual le permite entender el punto de vista del Estado colombiano sobre las competencias que los maestros deben saber para la enseñanza, que deben de hacer y saber cómo intelectuales reflexivos y cuáles contenidos pueden facilitar la comprensión de lo que sucede en cada momento histórico en un contexto mundial. Parte de las décadas de los años 60s donde la enseñanza de las Ciencias sociales se hace mediante guías, estaba determinada por una formación disciplinar centrada en la historia, geografía y cívica independientes entre sí, donde no reflejaba la relación entre los conocimientos del área social y la vida cotidiana del estudiante; narración de hechos, enseñados y evaluados desde la mecánica de la memorización. Los contenidos buscaban, la construcción de una identidad nacional. En la década de los 70s, los textos escolares de geografía e historia son resúmenes de las editoriales, como libros de trabajo, con talleres para los estudiantes, planeadores para los profesores, donde los contenidos y la planificación de la clase siguen siendo descriptivos. En los años 80s, se configura una conciencia política que hace del colombiano un sujeto social con conciencia y potencia que lo hace partícipe de sus decisiones. El movimiento pedagógico de los profesores de primaria y secundaria, exige un currículo participativo y crítico, con una formación basada en los avances de la pedagogía y la didáctica, pensada como su fundamento de su identidad y el saber propio de su profesión. En los años 90s, hay coincidencia en la integración de las Ciencias Sociales, reconociendo la realidad social compleja, desde saberes trasdisciplinares, 
enseñanza en grandes problemas y procesos sociales conectados con la realidad a partir de los aportes de la Nueva Historia.

La constitución política de 1991 y la Ley 115 de 1994, plantea la autonomía escolar mediante el Proyecto Educativo Institucional, según las necesidades de la región y del país. En la formación docente: la catedra afrocolombiana y el reconocimiento de una nación multicultural, multiétnico e intercultural. Se piensa la didáctica, desde una visión problematizadora de la enseñanza, a partir de lineamientos curriculares y en los estándares básicos en competencias; donde la didáctica de las Ciencias Sociales pasó de ser una instrumentación para la enseñanza a ser vista en Colombia y América Latina, como parte constitutiva de un proceso social. Finalmente, lo anterior le permite concluir que todo aspirante a Licenciado en Didáctica de las Ciencias Sociales debe saber: enseñar con una actitud histórica, espacial, con imaginación sociológica, con una visión etnográfica de la realidad social, con una actitud intercultural y ético-política. Desde esta perspectiva de pensar la Didáctica de las ciencias Sociales, es pensarla desde una postura de ciencia social en sí misma y se deja de lado la instrumentalización de la enseñanza, como fin último y como una teoría práctica no científica.

\section{Elaboró: William Pacheco Vargas}

Docente Escuela Ciencias Sociales

Grupo de investigación HISULA

\section{Misión Académica a Guinea Ecuatorial}

\section{1 a 15 de julio de 2016}

La Universidad Pedagógica y Tecnológica de Colombia, llega por primera vez al continente africano, a través del proyecto de investigación "Maestras indígenas,

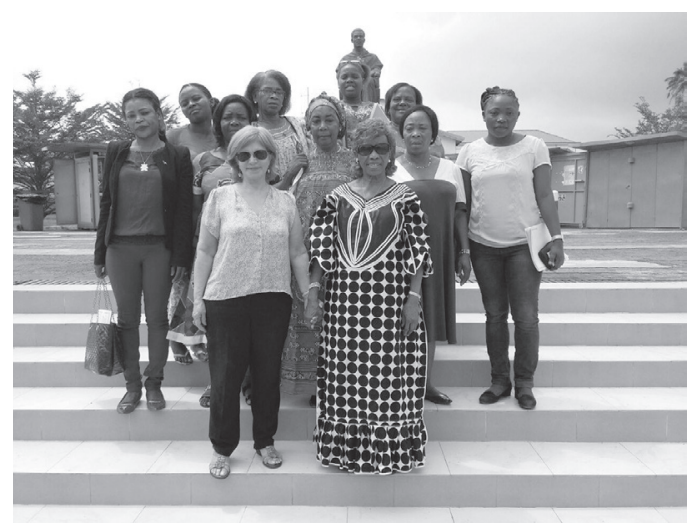
africanas y afrodescendientes", SGI de la DIN 1732. Este proyecto se enmarca dentro de la investigación, de Educadores Latinoamericanos, que presenta como resultados una colección de libros que van en el tomo VIII y es liderado por el Grupo de Investigación Historia y Prospectiva de la Universidad Latinoamericana. HISULA. El presente proyecto de investigación se vinculó en la 
República de Guinea Ecuatorial a través de la Facultad de Educación de la Universidad Nacional (UNGE) de este País. Asimismo, con el Viceministerio de Educación de Guinea Ecuatorial logrando establecer desde hace un año un grupo de docentes que son protagonistas en las historias de vida. Por otra parte, la Escuela Normal Superior de la República de Gabón se vinculó igualmente al proyecto. Precisamente, del 11 al 15 de julio de 2016, la Dra. Diana Elvira Soto Arango permaneció en Guinea Ecuatorial, realizando entrevistas a maestras ecuatoguineanas y gabonesas. Además, adelantó gestiones de convenios marcos para la Universidad Pedagógica y en el campo de la investigación. Destacamos el hecho de haber estructurado un artículo con investigadoras ecuatoguineanas con los resultados del citado proyecto de investigación; el haber concretado la venida de directivas e investigadoras de la UNGE y de Gabón al VI Congreso Internacional de Universidades y II Coloquio de Maestras indígenas, africanas y afrodescendientes, que se realizarán en el mes de octubre en Colombia (10 al 12) y Guatemala (17 a 19) y donde se firmarán los convenios que institucionalizarán los procesos conjuntos en investigaciones, movilidad, de estudiantes y profesores. Consideramos que con investigaciones como la de "Maestras indígenas, africanas y afrodescendientes" es que se permite un impacto social y la recuperación de nuestra cultura afrodescendiente que en los actuales momentos en Colombia es una aporte a la inclusión, la tolerancia que impulsamos desde la Facultad de Educación de la Universidad Pedagógica y Tecnológica de Colombia.

Elaboró: Diana Soto Arango Decana Facultad Ciencias de la Educación Grupo de investigación HISULA 


\section{Reconocimiento a Revistas Científicas de la Universidad Pedagógica y Tecnológica de Colombia}

\section{Tunja, 08 de agosto de 2016}

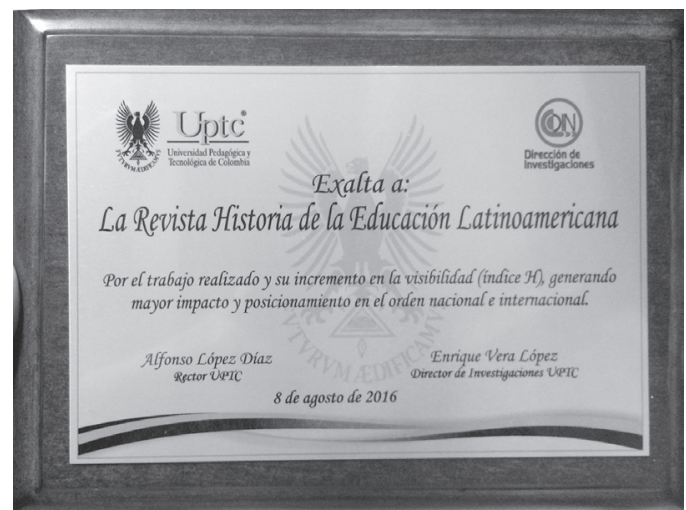

El pasado 8 de agosto de 2016 en las instalaciones de la Universidad Pedagógica y Tecnológica de Colombia, la Dirección de Investigaciones en cabeza del Dr. Enrique Vera, exalta a la Revista Historia de la Educación Latinoamericana, por el trabajo editorial realizado y por su incremento en el índice $\mathrm{H}$. Índice que genera mayor impacto de la publicación y la posiciona en el orden nacional e internacional como una revista de alta calidad académica y científica. 